\title{
Stochastic Poisson-Sigma model
}

\author{
RÉMI LÉANDRE
}

\begin{abstract}
We produce a stochastic regularization of the Poisson-Sigma model of Cattaneo-Felder, which is an analogue regularization of Klauder's stochastic regularization of the Hamiltonian path integral [23] in field theory. We perform also semi-classical limits.
\end{abstract}

Mathematics Subject Classification (2000): 53D55 (primary); 60G60, 60H07 (secondary).

\section{Introduction}

Let us consider a manifold $M$ endowed with a Poisson structure, a bilinear map \{., .\} from the space of smooth functions on the manifold into the space of smooth functions on the manifold, anticommutative and satisfying the Jacobi relation. Bayen, Flato, Fronsdal, Lichnerowicz and Sternheimer $[6,7]$ have introduced the so-called program of deformation quantization. These authors get the following formal series:

$$
f * g=\sum i^{n} h^{n} P_{n}(f, g) .
$$

The $P_{n}$ 's are differential operators. This series is diverging. The program of deformation quantization was carried out by Kontsevich [24]. We refer to the survey of Dito-Sternheimer about this topic [17].

Cattaneo-Felder [15] have established the link between Kontsevich formula and quantum field theory. Let us suppose that the manifold is $\mathbb{R}^{d}$. They consider the so-called Poisson-Sigma model. Let us recall how it is constructed: we consider the disk $D, 3$ points $\infty, 1,2$ on the boundary of the disk. They consider the space of forms $\eta$ on $D$ and the space of maps $X$ from $D$ into $\mathbb{R}^{d}$. Let $\alpha_{i, j}$ be the components of the Poisson structure on $\mathbb{R}^{d}$. Let $(t, s)=S$ be the polar coordinates on $D$ : $t \in[0,1], s \in S^{1}$. Cattaneo-Felder consider the action:

$$
\sum_{i} \int_{D} \eta_{i}(S) \wedge d X_{i}+\sum_{i, j} \int_{D} \alpha_{i, j}(X) \eta_{i} \wedge \eta_{j}=S(X, \eta)
$$

Pervenuto alla Redazione il 15 dicembre 2004 e in forma definitiva il 26 ottobre 2005. 
where $X=\left(X_{1}, \ldots, X_{d}\right)$ and where $\eta_{j}$ are 1-forms on $D$. [15] consider the following formula for the non-perturbative $*$-product:

$$
f *_{h} g(x)=\int_{\eta, X, X(\infty)=x} f(X(1)) g(X(2)) \exp [i S / h] d D X d D \eta
$$

where the field $X$ and the form $\eta$ are chosen at random according to the formal Lebesgue measure on the configuration space. [15] perform the semi-classical analysis when $h \rightarrow 0$ and get the asymptotic expansion:

$$
f *_{h} g(x)=\sum(i h)^{n} P_{n}(f, g) .
$$

The objects of Cattaneo-Felder are formal (see [20,21]) and use the heavy apparatus of quantum field theory. Our purpose is to add a stochastic regulator in (1.3) in order to define the functional integral rigorously. We get a stochastic product $f *_{s t, h} g$.

Let us recall that in (1.3), we have to choose two kinds of objects at random: the field $X: D \rightarrow \mathbb{R}^{d}$ and the forms $\eta$ over $D$. So we have to introduce stochastic regulators to define a random field $X$ and to define random forms $\eta$.

In order to define the random field $X: D \rightarrow \mathbb{R}^{d}$, we will follow the procedure of Airault-Malliavin [2]. Airault-Malliavin [2] have defined the Brownian motion over a loop group. Let us recall that infinite dimensional processes over infinite dimensional manifolds have a long history: see works of Kuo [26], BelopolskayaDaletskii [8] and Daletskii [16]. Albeverio-Léandre-Röckner [4] have defined the Ornstein-Uhlenbeck process over the free loop space, by using the theory of Dirichlet forms. Brzezniak-Elworthy [12] have given an abstract generalization of the works of Airault-Malliavin.

In this paper, we are concerned with a $(1+1)$-dimensional theory: this means we consider a diffusion process on the loop space. Various works in this direction were done by Brzezniak-Léandre [13, 14], Léandre [34, 35, 36]. Let us remark that in $(1.3)$, there is the condition $X(\infty)=x$. [14, 34, 36] have introduced a convenient Brownian bridge in order to do the conditional expectation by $X(\infty)=x$. But there is another procedure to condition functionals: it is the Airault-MalliavinSugita procedure $[1,44]$. In this work, we will follow this procedure.

In order to define random forms, we will employ the techniques of [37]. This means we will not choose our random forms on $D$ according to the formal Lebesgue measure on the space of forms, but we will introduce a stochastic Gaussian regulator in order to define the probability measure on the space of forms.

If we do not look at the conditional expectation by $X(\infty)=x$, the action $S$ becomes a stochastic integral, which belongs to all of the Sobolev spaces of the Malliavin Calculus [41]. We consider the measure

$$
h \rightarrow E[f(X(1)) g(X(2)) h(X(\infty)) \exp [i S]] .
$$

By Malliavin Calculus, it has a smooth density. Moreover, the magic properties of the Airault-Malliavin equation tell us that the density of the law of $X(\infty)$ is strictly positive. 
We have:

\section{Theorem A.}

$$
f *_{s t} g=E[f(X(1)) g(X(2)) \exp [i S] \mid X(\infty)=x]
$$

defines a continuous bilinear map from $C_{b}^{\infty}\left(\mathbb{R}^{d}\right)$ into $C_{b}^{\infty}\left(\mathbb{R}^{d}\right)$.

We use the Malliavin Calculus to prove Theorem A.

We perform a semi-classical analysis when $h \rightarrow 0$ : for that task, we choose a small leading Brownian motion as well as a small stochastic regularization of $\eta$. Such considerations were done in [32]. But $S$ is only a stochastic integral: so, by improving a bit the techniques of [32], we have:

\section{Theorem B.}

$$
f *_{s t, h} g=E_{h}[f(X(1)) g(X(2)) \exp [i S / h] \mid X(\infty)=x]
$$

has, when $h \rightarrow 0$ an asymptotic expansion:

$$
f *_{s t, h} g=\sum h^{n} Q_{n}(f, g)
$$

where the $Q_{n}$ 's are differential operators acting on $f$ and $g$.

For that, we use the techniques of asymptotics of Wiener functionals by using the Malliavin Calculus: we refer to the surveys by Léandre [28], Kusuoka [27] and Watanabe [45] for this topic.

The reader interested in the relation existing between analysis over loop space and mathematical physics can consult the survey by Albeverio [3] and the two surveys by Léandre [29, 30].

\section{The model without conditioning}

Let $\Pi(x)$ be a linear map from $\mathbb{R}^{n}$ into $\mathbb{R}^{d}$, which depends smoothly from $x \in \mathbb{R}^{d}$ : we suppose that the derivatives of all orders of $\Pi$ are bounded and that $\left(\Pi(x) e_{i}\right)$ $i=1, \ldots, n$ spans uniformly $\mathbb{R}^{d}$ for the canonical basis $e_{1}, \ldots, e_{n}$ of $\mathbb{R}^{n}$.

Let $H=H^{1,2}\left(S^{1} ; \mathbb{R}^{n}\right)$ be the Hilbert space of maps from the circle $S^{1}$ into $\mathbb{R}^{n}$ such as:

$$
\int_{0}^{1}|h(s)|^{2} d s+\int_{0}^{1}|d / d s h(s)|^{2} d s=\|h\|^{2}<\infty .
$$

We write $h=\left(h^{1}, \ldots, h^{n}\right)$. Moreover,

$$
h^{j}(0)=\left\langle h, e^{j}\right\rangle
$$


where

$$
e^{j}(s)=(0, \ldots, 0, \lambda \exp [-s]+\mu \exp [s], 0, \ldots, 0)
$$

for some $\lambda$ and some $\mu$ for $0 \leq s \leq 1$. Moreover $e^{j}(s)$ is smooth on ]0, 1 [ with half derivatives at all orders at 0 and $1: e^{j}(0)=e^{j}(1)$ but $d / d s e^{j}(0) \neq d / d s e^{j}(1)$.

We have:

$$
h^{j}(s)=\left\langle h, e^{j}(.-s)\right\rangle .
$$

We consider the Brownian motion with values in $H$ :

$$
B_{t}(s)=\left(B_{t}^{1}(s), \ldots, B_{t}^{n}(s)\right) .
$$

The processes $B^{j}($.$) are independent and t \rightarrow B_{t}^{j}(s)$ is a Brownian motion with values in $\mathbb{R}$ submitted to the relation:

$$
d\left\langle B_{t}^{j}(s), B_{t}^{j}\left(s^{\prime}\right)\right\rangle=e\left(s-s^{\prime}\right) d t
$$

where $e^{j}(s)$ is the $j^{\text {th }}$ coordinate of $e(s)$.

We consider the Airault-Malliavin equation [2, 12]:

$$
d x_{t}(s)(x)=\Pi\left(x_{t}(s)(x)\right) d_{t} B_{t}(s) ; x_{0}(s)(x)=x .
$$

It is a family of Stratonovitch equations. We have shown that $s \rightarrow x_{t}(s)(x)$ is $1 / 2-\epsilon$ Hölder by Gronwall lemma and Kolmogorov lemma [39]: we have an improvement of this result. Namely:

Proposition 2.1. $x \rightarrow\left(s \rightarrow x_{1}(s)(x)\right)$ is almost-surely smooth for the Hölder topology.

Proof. This comes from the fact that $s \rightarrow \frac{D^{(r)}}{D x^{(r)}} x_{1}(s)(x)$ is almost surely Hölder $1 / 2-\epsilon$ in $s$ (see [32] for an analogous statement). Namely, the stochastic differential equation of $\frac{D}{D x} x_{t}(s)(x)$ is

$$
d \frac{D}{D(x)} x_{t}(s)(x)=D \Pi\left(x_{t}(s)(x)\right) \frac{D}{D(x)} x_{t}(s)(x) d_{t} B_{t}(s)
$$

and we get by induction the differential equation of $\frac{D^{(r)}}{D x^{(r)}} x_{t}(s)(x)$.

Let us write for $\Delta s$ small:

$$
B_{t}(s+\Delta s)=B_{t}(s)+\Delta_{s} B_{t}(s) .
$$


We have (See [35] Part III):

Property H(1). If $s^{\prime}$ does not belong to $] s, s+\Delta s[$ :

$$
d\left\langle\Delta_{s} B .(s), B .\left(s^{\prime}\right)\right\rangle=d t C\left(s, s^{\prime}\right) \Delta s+O\left(\Delta s^{2}\right) .
$$

Property H(2). if $] s, s+\Delta s[\cap] s^{\prime}, s^{\prime}+\Delta s^{\prime}[=\emptyset$, we have:

$$
d\left\langle\Delta_{s} B .(s), \Delta_{s^{\prime}} B .\left(s^{\prime}\right)\right\rangle=d t C\left(s, s^{\prime}\right) \Delta s \Delta s^{\prime}+O\left(\Delta s+\Delta s^{\prime}\right)^{3} .
$$

Let us consider a sequence of intervals $] s_{i}, s_{i}+\Delta s_{i}$ [, two intervals being either disjoint or equal. We denote by $|I|$ the number of intervals and by $\|I\|$ the number of distinct intervals. Let us consider some points $r_{j}$ of the circle which do not belong to the union of the previous open intervals. Let $\alpha_{t}(i)$ be some processes, which are $B .\left(r_{j}\right)$ measurable, previsible, and which are semi-martingales. We suppose that the local characteristic [22] of each $\alpha_{t}(i)$ have bounded Sobolev norms in the sense of the Malliavin Calculus [41] for the Gaussian space spanned by the $B .\left(r_{j}\right)$. We put iteratively:

$$
I^{i+1}(t)=\int_{0}^{t} I^{i}(u) \alpha_{u}(i) d_{u} \Delta_{s_{i}} B_{u}\left(s_{i}\right)
$$

and we get an iterated Stratonovitch integral $I^{I}(t)$. Let $F$ be a measurable functional for the Gaussian space spanned by the $B .\left(r_{j}\right)$ : we suppose that $F$ has bounded Sobolev norms in the sense of Malliavin Calculus for the space spanned by the $B .\left(r_{j}\right)$. We denote by $I^{\prime}$ the set of indices obtained by selecting from $I$ an interval only one time. The cardinal of $I^{\prime}$ is therefore $\|I\|$.We have the main lemma:

\section{Lemma 2.2.}

$$
E\left[F I^{I}(t)\right] \leq C \prod_{i \in I^{\prime}} \Delta s_{i}
$$

where $C$ can be estimated in terms of the Sobolev norms of $F$ and of the $\alpha .(i)$.

Proof. We apply the Clark-Ocone formula to $F$ [41]. We select the Itô term in $I^{I}(t)$ and the finite energy term in $I^{I}(t)$. We conclude by applying Itô formula and Properties $\mathrm{H}(1)$ and $\mathrm{H}(2)$ and property $\mathrm{H}(3)$ :

\section{Property H(3).}

$$
d_{t}\left\langle\Delta_{s} B .(s), \Delta_{s} B .(s)\right\rangle=C(s) \Delta s d t+O\left(\Delta s^{2}\right) d t .
$$

The statement follows by induction on $|I|$.

Remark 2.3. We remark that we have analogue estimates if we consider a product $\prod_{i \in I} I^{i}(t)$ of single integrals or if we consider double iterated integrals in the 
product. Namely, we can come back to the situation of Lemma 2.2 by using the Itô-Stratonovitch formula. We put:

$I_{t}^{1}(s, \Delta s)(x)=\frac{D}{D(x)} x_{t}(s)(x) \int_{0}^{t} D \Pi\left(x_{u}(s)(x)\right) \frac{D^{-1}}{D(x)} x_{u}(s)(x) d_{u} \Delta_{s} B_{u}(s)$

and

$I_{t}^{2}(s, \Delta s)(x)$

$=\frac{D}{D(x)} x_{t}(s)(x) \int_{0}^{t} \frac{D^{-1}}{D(x)} x_{u}(s)(x)\left\langle D \Pi\left(x_{u}(s)(x)\right), I_{u}^{1}(s, \Delta s)(x), d_{u} \Delta_{s} B_{u}(s)\right\rangle$.

By using the rule of differentiation of stochastic differential equations along a parameter [10, 25], we have that:

$$
x_{t}(s+\Delta s)(x)=x_{t}(s)(x)+I_{t}^{1}(s, \Delta s)(x)+I_{t}^{2}(s, \Delta s)(x)+O\left(\Delta s^{3 / 2}\right) .
$$

The error term is uniform in $x$ over each compact set of $\mathbb{R}^{d}$.

Let us consider a 1 -form on $[0,1] \times S^{1}, \eta=\eta_{1} d s+\eta_{2} d t$. We put a Gaussian measure on the set of $\eta: \eta_{1}$ and $\eta_{2}$ are independent. On the space of $\eta$ we consider a Gaussian measure whose reproducing Hilbert space is defined as follows: we consider the space of function taking values in $\mathbb{R}^{2 d}$ endowed with the Sobolev norm $\int_{S^{1}}\left\langle\left(-\frac{d^{2}}{d s^{2}}+1\right) \eta(s), \eta(s)\right\rangle d s=\|\eta\|_{H^{d}}^{2}$ and the space of forms endowed with the Hilbert norm $\int_{0}^{1}\left\|\frac{\partial}{\partial t} \eta_{t}(.)\right\|_{H^{d}}^{2} d t$. The random forms which are obtained in that way are almost surely Hölder. Let us consider $N=2^{N_{0}}$. We consider the polygonal approximation $s \rightarrow x_{t}^{N}(s)(x)$ of $s \rightarrow x_{t}(s)(x)$. We consider a coordinate $x^{N, j}(s)(x)$ of it. We put:

$$
A_{t}^{N, j}(x)=\int_{S^{1}} \eta_{2}^{j}(s, t) d_{s} x_{t}^{N, j}(s)(x)
$$

We have:

Proposition 2.4. When $N \rightarrow \infty, A_{t}^{N, j}(x)$ tends in all of the $L^{p}$ to a real random variable

$$
\int_{S^{1}} \eta_{2}^{j}(s, t) d_{s} x_{t}^{j}(s)(x) .
$$

Moreover, the stochastic integral defined in (2.19) depends almost surely smoothly on $x$ and in all of the $L^{p}$.

Proof. We omit to write the index $j$, doing as if the diffusion $x_{t}(s)(x)$ was one dimensional. We write:

$$
A_{t}^{N}(x)=\sum A_{i}^{N}=\sum \int_{s_{i}}^{s_{i+1}} \eta_{2}(s, t) d_{s} x_{t}^{N}(s)(x) .
$$


Let us decompose $A_{i}^{N}$ as a sum:

$$
A_{i}^{N}=B_{i}^{N}+C_{i}^{N}
$$

where

$$
B_{i}^{N}=\eta_{2}\left(s_{i}, t\right) \Delta_{s_{i}} x_{t}\left(s_{i}\right)(x)
$$

and where:

$$
C_{i}^{N}=\int_{s_{i}}^{s_{i+1}}\left(\eta_{2}(s, t)-\eta_{2}\left(s_{i}, t\right)\right) \frac{d s}{s_{i+1}-s_{i}} \Delta_{s_{i}} x_{t}\left(s_{i}\right)(x) .
$$

First step. Convergence of $\sum B_{i}^{N}$ in all the $L^{p}$.

We write $\Delta s_{i}=1 / N$. Moreover,

$$
\begin{aligned}
B_{i}^{N}=B_{i, 1}^{N} & +B_{i, 2}^{N}+\text { error } \\
& =\eta_{2}\left(s_{i}, t\right) I_{t}^{1}\left(s_{i}, \Delta s_{i}\right)(x)+\eta_{2}\left(s_{i}, t\right) I_{t}^{2}\left(s_{i}, \Delta s_{i}\right)(x)+\text { error } .
\end{aligned}
$$

Let us study first the convergence of $\sum B_{i, 1}^{N}$ in all the $L^{p}$. Let $N^{\prime}=2^{N_{0}^{\prime}}$ be an integer larger than $N$. We write:

$$
D_{i}^{N}=B_{i, 1}^{N}-\sum_{\left[s_{i^{\prime}}, s_{i^{\prime}+1}\right] \subseteq\left[s_{i}, s_{i+1}\right]} B_{i^{\prime}, 1}^{N^{\prime}} .
$$

In $B_{i, 1}^{N}$ and in $I_{t}^{1}\left(s_{i}, \Delta s_{i}\right)$, we get:

$$
d_{t} \Delta_{s_{i}} B_{t}\left(s_{i}\right)=\sum_{\left[s_{i^{\prime}}, S_{i^{\prime}+1}\right] \subseteq\left[s_{i}, s_{i+1}\right]} d_{t} \Delta_{s_{i^{\prime}}} B_{t}\left(s_{i^{\prime}}\right)
$$

and we apply Lemma 2.2 in order to get the estimate:

$$
E\left[\prod_{i_{j} \in I} D_{i_{j}}^{N}\right]=o(1) \prod_{i_{j} \in I^{\prime}} \Delta s_{i_{j}}=o(1) C(I) .
$$

But there are at most $C N^{r}$ set of multi-indices $I$ such that $|I|=p$ and $\|I\|=r$. Therefore the result.

Let us study the behaviour of $\sum B_{i, 2}^{N}$ in (2.24).

In $I_{t}^{2}\left(s_{i}, \Delta s_{i}\right)$, we write:

$$
d_{u} \Delta_{s_{i}} B_{u}\left(s_{i}\right) d_{v} \Delta_{s_{i}} B_{v}\left(s_{i}\right)=\sum_{\left[s_{j}, s_{j+1}\right],\left[s_{j^{\prime}}, s_{j^{\prime}+1}\right] \subseteq\left[s_{i}, s_{i+1}\right]} d_{u} \Delta_{s_{j}} B_{u}\left(s_{j}\right) d_{v} \Delta_{s_{j^{\prime}}} B_{v}\left(s_{j^{\prime}}\right) .
$$


We select in the decomposition (2.27) the sum where we have $s_{j}=s_{j^{\prime}}$, and we get a decomposition of $B_{i, 2}^{N}$ into $D_{i, 1}^{N}+D_{i, 2}^{N}$ where in $D_{i, 1}^{N}=\sum D_{i, j, 1}^{N}$ we consider only the diagonal terms.

We write:

$$
\begin{aligned}
& \sum \eta_{2}\left(s_{i}, t\right) D_{i, 1}^{N}-\sum \eta_{2}\left(s_{j}, t\right) B_{j, 2}^{N^{\prime}} \\
&=\sum_{\left[s_{j}, s_{j+1}\right] \subseteq\left[s_{i}, s_{i+1}\right]}\left(\eta_{2}\left(s_{i}, t\right)-\eta_{2}\left(s_{j}, t\right)\right) D_{i, j, 1}^{N}+ \\
& \sum_{\left[s_{j}, s_{j+1}\right] \subseteq\left[s_{i}, s_{i+1}\right]} \eta_{2}\left(s_{j}, t\right)\left(D_{i, j, 1}^{N}-B_{j, 2}^{N^{\prime}}\right) .
\end{aligned}
$$

The first term tends trivially to 0 in all of the $L^{p}$. By applying the remark following Lemma 2.2, the second term tends to zero in all of the $L^{p}$ when $N^{\prime} \rightarrow \infty$.

Let us consider $\sum \eta_{2}\left(s_{i}, t\right) D_{i, 2}^{N}$. Let us show that it tends in all the $L^{p}$ to zero. Let $I=\left\{i_{1}, \ldots, i_{|I|}\right\}$ with $\|I\|$ given. According to Lemma 2.2. we have:

$$
E\left[\prod_{i_{j} \in I} D_{i, 2}^{N}\right]=O\left(N^{-\|I\|}\right) .
$$

Hence, we can write $D_{i, 2}^{N}=\sum_{j \neq j^{\prime}} D_{j, j^{\prime}, 2}^{N}$. If we distribute in $\left(D_{i, 2}^{N}\right)^{r}$, there are at most $C\left(N^{\prime} / N\right)^{k}$ products $\prod D_{j_{l}, j_{l^{\prime}}, 2}^{N}$ where the cardinal described by $j_{l}, j_{l^{\prime}}$ is $k$. But $k$ is at least equal to 2. Therefore (2.30). We conclude as in (2.27).

Second step. Convergence of $\sum C_{i}^{N}$ in all of the $L^{p}$.

We write

$$
\sum C_{i}^{N}=\sum \alpha^{N}\left(s_{i}\right) I_{t}^{1}\left(s_{i}, \Delta s_{i}\right)(x)+\text { error }
$$

where $\alpha^{N}\left(s_{i}\right)$ is independent of the system of $I_{t}^{1}\left(s_{i}, \Delta s_{i}\right)$ and tends to 0 in all of the $L^{p}$. Therefore the sum tends to 0 by the previous considerations in all the $L^{p}$.

In order to show that the stochastic integral defined by (2.19) depends almost surely and in all of the $L^{p}$ from $x$, we can apply the previous considerations to $\frac{D^{r}}{D x^{r}} A_{t}^{N, j}(x)$ and show that it converges in all the $L^{p}$ to $\frac{D^{r}}{D x^{r}} \int_{S^{1}} \eta_{2}^{j}(s, t) d_{s} x_{t}^{j}(s)(x)$. The Sobolev imbedding theorem allows to conclude.

Let us introduce the stochastic Poisson-Sigma action defined as follows:

$$
\begin{aligned}
S(x .(.)(x), \eta)= & \sum_{j} \int_{[0,1] \times S^{1}} \eta^{j} \wedge d x_{.}^{j}(.)(x) \\
& +\sum_{i, j} \int_{[0,1] \times S^{1}} \alpha_{i, j}(x .(.)(x)) \eta^{i} \wedge \eta^{j}
\end{aligned}
$$


Proposition 2.4 allows us to deduce the following theorem:

Theorem 2.5. The random variable $S(x .().(x), \eta)$ is real, and is almost surely differentiable in $x$. For all $r$, all $p \geq 1$ :

$$
\sup _{x} E\left[\left|\frac{D^{r}}{D x^{r}} S\left(\eta_{.}, x_{.}(.)(x)\right)\right|^{p}\right]<\infty \text {. }
$$

This allows us to state the following theorem: let $C_{b}^{\infty}\left(\mathbb{R}^{d}\right)$ be the Fréchet space of smooth functions $f$ on $\mathbb{R}^{d}$ with bounded derivatives at each order endowed with the set of semi-norms:

$$
\|f\|_{r, \infty}=\sup _{x}\left|\frac{D^{r}}{D x^{r}} f(x)\right|
$$

Theorem 2.6. The map which sends $(f, g)$ to:

$$
\left.E\left[f\left(x_{1}(1)(x)\right) g\left(x_{1}(2)(x)\right) \exp [i S(x .(.)(x)), \eta)\right]\right]
$$

is a continuous bilinear application from $C_{b}^{\infty}\left(\mathbb{R}^{d}\right)$ into $C_{b}^{\infty}\left(\mathbb{R}^{d}\right) .1$ and 2 denote in (2.35) two different points of $S^{1}$.

\section{A stochastic star product}

Let us recall that, if the Malliavin Calculus has a lot of precursors (see the work of Hida, Elworthy, Fomin, Albeverio ... ), the main novelty of the Malliavin Calculus was to complete the differential operations known at that time on the Wiener space in all of the $L^{p}$. This allowed Malliavin to recover Hörmander's theorem by probabilistic methods [38]. The first ones who have applied the Malliavin Calculus to other Gaussian spaces than the traditional Wiener space are Nualart and Sanz [42] in order to study the Brownian sheet. Here, we apply the Malliavin Calculus in our situation.

We consider the space $H(B)$ of maps from $[0,1]$ into $H, h_{t}().(B)$, such that

$$
\int_{0}^{1}\left\|\frac{\partial}{\partial t} h_{t}(.)(B)\right\|_{B}^{2} d t<\infty
$$

and the space $H(\eta)$ of maps from $[0,1]$ into $H^{d}, h_{t}().(\eta)$, such that

$$
\int_{0}^{1}\left\|\frac{\partial}{\partial t} h_{t}(.)(\eta)\right\|_{H^{d}}^{2} d t<\infty .
$$

$H(B)$ is the Hilbert reproducing space of the Gaussian field $B$.(.) and $H(\eta)$ is the Hilbert reproducing space of the Gaussian field $\eta$. 
If $F$ is a functional which is $B$.(.) and $\eta$ measurable, we take its derivative in the direction of $H(B)$ and $H(\eta) . \nabla^{r} F$ is therefore a random element of $(H(B) \oplus$ $H(\eta))^{\otimes r}$. We consider its $L^{p}$ norm and we get:

$$
\|F\|_{r, p}=E\left[\left\|\nabla^{r} F\right\|^{p}\right]^{1 / p}
$$

which is the collection of Sobolev norms in the sense of the Malliavin Calculus [41]. $F$ is said to be smooth in the Malliavin sense if $\|F\|_{r, p}<\infty$ for all $r$ and $p$.

Lemma 3.1. $\frac{D^{r}}{D x^{r}} x_{t}(s)(x)$ and $\frac{D^{-1}}{D x} x_{t}(s)(x)$ are smooth in the sense of Malliavin. Moreover their Sobolev norms are bounded in $s, t \in[0,1]$ and $x$, and the kernel of their derivatives are B. (s)- measurable.

Proof. This result is classical [41] if we consider these functionals as $B$.(s)-measurable. But

$$
d / d t h_{t}(s)(B)=d / d t\left\langle h_{t}(.)(B), e(.-s)\right\rangle .
$$

Therefore the result.

Proposition 3.2. $\frac{D^{r}}{D x^{r}} A_{t}^{N, j}(x)$ tends to $\frac{D^{r}}{D x^{r}} \int_{S^{1}} \eta_{2}^{j}(s, t) d_{s} x_{t}^{j}(s)(x)$ in all the Sobolev spaces and the Sobolev norms of this last stochastic integral are bounded in $x \in R^{d}$.

Proof. If we do not take the derivatives of $d_{u} \Delta_{s} B_{u}(s)$ and $d_{v} \Delta_{s} B_{v}(s) d_{u} \Delta_{s} B_{u}(s)$ in (2.15) and in (2.16), the result goes by the same methods as the proof of Proposition 2.4, by applying Lemma 3.1. Let us take the derivatives of $d_{u} \Delta_{s} B_{u}(s)$ in (2.15) and (2.16). They are given by $\frac{\partial}{\partial u} \Delta_{s} h_{u}(s)(B)=\frac{\partial}{\partial u}\left\langle h_{u}(.)(B), e(.-s-\Delta s)-e(.-s)\right\rangle_{H}$ and therefore the treatment leads to simpler considerations than in the statement of Proposition 2.4.

We deduce from Proposition 3.2 that $\frac{D^{r}}{D x^{r}} S(x .().(x), \eta)$ is bounded in $x$ in all the Sobolev spaces. We get, since the stochastic Poisson-Sigma action $S(x .().(x), \eta)$ is real, that:

Proposition 3.3. Let $\mu(x)$ be the measure on $R^{d}$ which sends $h \in C_{b}\left(\mathbb{R}^{d}\right)$ to:

$$
\left.E\left[f\left(x_{1}(1)(x)\right) g\left(x_{1}(2)(x)\right) h\left(x_{1}(\infty)(x)\right) \exp [i S(x .(.)(x)), \eta)\right]\right]
$$

where $f$ and $g$ belong to $C_{b}^{\infty}\left(\mathbb{R}^{d}\right) . \mu(x)$ has a density $q(x, y)$ with respect to the Lebesgue measure and the uniform norm of $\frac{D^{r}}{D x^{r}} \frac{D^{r^{\prime}}}{D y^{r^{\prime}}} q(x, y)$ can be estimated in terms of the uniform norms of the derivatives of $f$ and $g$.

Proof. This comes from the fact that $\frac{D^{r}}{D x^{r}} \exp [i S(x .().(x), \eta)]$ and $\frac{D^{r}}{D x^{r}} x_{1}(s)(x)$ have bounded Sobolev norms in the sense of the Malliavin Calculus in $x$ and from the Malliavin Calculus [41]. 
Proof of Theorem A. $x_{1}(\infty)(x)$ is given by a diffusion on $\mathbb{R}^{d}$. Its law has a smooth density $p_{1}(x, y)>0$ with bounded derivatives of all orders in $x$ and $y$. By using the Airault-Malliavin-Sugita procedure [1, 44], we get :

$$
\frac{\mu(x, x)}{p_{1}(x, x)}=E\left[f\left(x_{1}(1)(x)\right) g\left(x_{1}(2)(x)\right) \exp [i S(x .(.)(x), \eta)] \mid x_{1}(\infty)(x)=x\right] \text {. }
$$

Then the result follows, since $p_{1}(x, x)>c>0$.

\section{Semi-classical analysis}

Following [40] and [18], let us put $\epsilon=h^{1 / 2}$. We replace $B$.(.) by $\epsilon B$.(.) and $\eta$ by $\epsilon \eta$. We get a random field $x .().(\epsilon)(x)$.

By using the classical rules of differentiation of $x_{t}(s)(\epsilon)(x)$ along the parameter $\epsilon$ and $x[10,39,25]$ and considerations analog to Lemma 3.1, we get:

Lemma 4.1. $\frac{D^{r^{\prime}}}{D \epsilon^{r^{\prime}}} \frac{D^{r}}{D x^{r}} x_{t}(s)(\epsilon)(x)$ and $\frac{D^{r^{\prime}}}{D \epsilon^{r^{\prime}}} \frac{D^{-1}}{D x} x_{t}(s)(\epsilon)(x)$ are smooth in the sense of Malliavin for the total Gaussian space. Moreover, their Sobolev norms are bounded in $s, t \in[0,1], \epsilon \in[0,1]$ and $x$ in $\mathbb{R}^{d}$ and the kernels of their derivatives are $B .(s)$-measurable.

We get by adding the new parameter $\epsilon$ :

Proposition 4.2. $\frac{D^{r^{\prime}}}{D \epsilon^{r^{\prime}}} \frac{D^{r}}{D x^{r}} A_{t}^{N, j}(\epsilon)(x)$ tends to $\left.\frac{D^{r^{\prime}}}{D \epsilon^{r^{\prime}}} \frac{D^{r}}{D x^{r}} \int_{S^{1}} \epsilon \eta_{2}^{j}(s, t) d_{s} x_{t}^{j}(s)(\epsilon)(x)\right)$ in all of the Sobolev spaces of the Malliavin Calculus. The Sobolev norms in the sense of Malliavin Calculus of the last stochastic integral are bounded in $x \in \mathbb{R}^{d}$ and $\epsilon \in[0,1]$. Moreover, they are 0 if $r^{\prime}=0$ or $r^{\prime}=1$.

We get:

Proposition 4.3. Let $\mu_{\epsilon}(x)$ be the measure on $\mathbb{R}^{d}$ which to $h \in C_{b}\left(\mathbb{R}^{d}\right)$ assigns:

$$
\left.E\left[f\left(x_{1}(1)(\epsilon)(x)\right) g\left(x_{1}(2)(\epsilon)(x)\right) \exp \left[i / \epsilon^{2} S(x .(.)(\epsilon)(x)), \epsilon \eta\right)\right] h\left(x_{1}(\infty)(\epsilon)(x)\right)\right]
$$

where $f$ and $g$ belong to $C_{b}^{\infty}\left(\mathbb{R}^{d}\right) . \mu_{\epsilon}(x)$ has a density $q_{\epsilon}(x)(\epsilon>0)$ and when $\epsilon \rightarrow 0$ :

$$
q_{\epsilon}(x, x)=\epsilon^{-d} \sum_{i=1}^{n} h^{i} \tilde{Q}_{i}(f, g)(x)+O\left(h^{n}\right)
$$

where $\tilde{Q}_{i}$ are differential operators in $f$ and $g$. 
Proof. $q_{\epsilon}(x, x)=\epsilon^{-d} \tilde{q}_{\epsilon}(x, 0)$ where $\tilde{q}_{\epsilon}(x, y)$ is the density of the measure $v_{\epsilon}$ :

$E\left[f\left(x_{1}(1)(\epsilon)(x)\right) g\left(x_{1}(2)(\epsilon)(x)\right) \exp \left[i / \epsilon^{2} S((x .().(\epsilon)(x), \epsilon \eta)] h\left(\frac{x_{1}(\infty)(\epsilon)(x)-x}{\epsilon}\right)\right]\right.$.

The result follows by standard arguments of Malliavin Calculus depending on a parameter [28, 27, 45] because, in all the Sobolev spaces of Malliavin Calculus, when $\epsilon \rightarrow 0, x_{1}(1)(\epsilon)(x) \rightarrow x, x_{1}(2)(\epsilon)(x) \rightarrow x, \frac{x_{1}(\infty)(\epsilon)(x)-x}{\epsilon}$ tends to the nondegenerate Gaussian variable $\Pi(x) B_{1}(\infty)$ and

$$
\epsilon^{-2} S(x .(.)(\epsilon)(x), \epsilon \eta) \rightarrow \sum_{i, j} \alpha_{i, j}(x) \int_{D} \eta_{i} \wedge \eta_{j}+\sum_{i} \int_{D} \eta_{i} \wedge d B_{i} .
$$

Let us write the measure $v_{\epsilon}$

$$
h \rightarrow E\left[G_{\epsilon} h\left(Z_{\epsilon}\right)\right]
$$

$G_{\epsilon}$ depends smoothly on $\epsilon$ in all of the Sobolev spaces of the Malliavin Calculus as well as $Z_{\epsilon}$. Moreover $Z_{\epsilon}$ satisfies uniformly in $\epsilon$ Malliavin's nondegeneracy condition: $\sup _{\epsilon} E\left[\left\langle\nabla Z_{\epsilon}, \nabla Z_{\epsilon}\right\rangle^{-p}\right]<\infty$ for all positive integers $p$.

We have:

$$
\frac{d^{r}}{d \epsilon^{r}} v_{\epsilon} h=\sum_{\left|\left(r^{\prime}\right)\right| \leq r} E\left[\tilde{G}_{\left(r^{\prime}\right)}(\epsilon) \frac{\partial^{\left(r^{\prime}\right)}}{\partial y^{\left(r^{\prime}\right)}} h\left(Z_{\epsilon}\right)\right]
$$

But by Malliavin's condition of nondegeneracy, we can remove the derivative of $h$ in (4.6) and we get

$$
\frac{d^{r}}{d \epsilon^{r}} v_{\epsilon} f=E\left[\bar{G}_{r}(\epsilon) h\left(Z_{\epsilon}\right)\right]
$$

Therefore $\tilde{q}(x, 0)$ is smooth in $\epsilon$.

At $\epsilon=0$, the Malliavin matrix of the Gaussian $Z_{0}$ is deterministic and $\bar{G}_{r}(0)$ contains only expressions in the Gaussian terms which are of the same parity as $r$. If $r$ is odd

$$
E\left[\bar{G}_{r}(0) \mid \Pi(x) B_{1}(\infty)=0\right]=0
$$

because we consider centered Gaussian variables. $\tilde{q}_{\epsilon}(x, 0)$ has therefore an asymptotic expansion $\sum \epsilon^{i} \bar{Q}_{i}(f, g)(x)$ but only the even powers of $\epsilon$ remain in this asymptotic expansion: namely the odd exponent leads to the expectation of odd functionals of some centered Gaussian measures, which are 0 . The introduction of derivatives of $f$ and $g$ is due to the asymptotic expansion of $f\left(x_{1}(1)(\epsilon)(x)\right)$ and of $g\left(x_{1}(2)(\epsilon)(x)\right)$ when $\epsilon \rightarrow 0$ because $x_{1}(1)(\epsilon)(x)$ and $x_{1}(2)(\epsilon)(x)$ go to $x$ in all the Sobolev spaces of the Malliavin Calculus when $\epsilon \rightarrow 0$. 
On the other hand, $p_{\epsilon}(x, x)$ has an asymptotic expansion:

$$
p_{\epsilon}(x, x)=\epsilon^{-d} \sum_{i=1}^{n} h^{i} c_{i}(x)+O\left(h^{n}\right)
$$

where the coefficients belong to $C_{b}^{\infty}\left(\mathbb{R}^{d}\right)$ and $c_{0}(x)>c>0$.

Proof of theorem B. We get

$$
f *_{s t, h} g(x)=\frac{\sum h^{i} \tilde{Q}_{i}(f, g)(x)+O\left(h^{n}\right)}{\sum_{i=0}^{n} h^{i} c_{i}(x)+O\left(h^{n}\right)} .
$$

The result holds because $c_{0}(x)>c>0$.

\section{References}

[1] H. Airault and P. Malliavin, "Quasi-sure Analysis", Publication Université Paris VI, Paris, 1990.

[2] H. Airault and P. Malliavin, "Integration on Loop Groups", Publication Université Paris VI, Paris, 1990.

[3] S. AlbEVERIO, Loop groups, random gauge fields, Chern-Simons models, strings: some recent mathematical developments, In: "Espaces de Lacets", R. Léandre, S. Paycha and T. Wurzbacher (eds.), Publi. Univ. Strasbourg, Strasbourg, 1996, 5-34.

[4] S. Albeverio, R. LÉANDre and M. RöCKNer, Construction of a rotational invariant diffusion on the free loop space. C.R. Acad. Sci. Paris Sér. I Math. 316 (1993), 287-292.

[5] M. ARnAUdon and S. PAYCHA, Stochastic tools on Hilbert manifolds: interplay with geometry and physics, Comm. Math. Phys. 197 (1997), 243-260.

[6] F. Bayen, M. Flato, C. Fronsdal, A. Lichnerowicz and D. Sternheimer, Deformation theory and quantization, I. Ann. Phys. (NY) 111 (1978), 61-110.

[7] F. Bayen, M. Flato, C. Fronsdal, A. Lichnerowicz and D. Sternheimer, Deformation quantization and quantization, II. Ann. Phys. (NY) 111 (1978), 111-151.

[8] Y. Belopolskaya and Y. K. DaletskiI, "Stochastic Equations and Differential Geometry", Kluwer, Dordrecht, 1990.

[9] Y. BelOPOLSKAYA and Y. GlikLiKh, Stochastic processes on groups of diffeomorphisms and viscous hydrodynamics, Inf. Dimens. Anal. Quantum Probab. Relat. Top. 5 (2002), 145159.

[10] J. M. Bismut, "Mécanique Aléatoire”, Lect. Notes. Math., Vol. 966, Springer, Heidelberg, 1981.

[11] J. M. Bismut, "Large Deviations and Malliavin Calculus", Progress in Math., Vol. 45, Birkhäuser, Basel, 1984.

[12] Z. BRZEZniAK and K. D. Elworthy, Stochastic differential equations on Banach manifolds, Method. Funct. Anal. Topology 6 (in honour of Y. Daletskii) (2000), 43-84.

[13] Z. BRZEZNIAK and R. LÉANDRE, Horizontal lift of an infinite dimensional diffusion, Potential Anal. 12 (2000), 249-280.

[14] Z. BRZEZNIAK and R. LÉANDRE, Brownian pants on a manifold, Preprint.

[15] A. CatTANeO and G. FeldeR, A path integral approach to Kontsevich quantization formula, Comm. Math. Phys. 212 (2000), 591-611.

[16] Y. DALETSKII, Measures and stochastic equations on infinite-dimensional manifolds, In: "Espaces de Lacets", R. Léandre, S. Paycha and T. Wuerzbacher (eds.), Publi. Univ. Strasbourg, Strasbourg, 1996, 45-52. 
[17] G. Dito and D. STERnheIMER, Deformation quantization; genesis, developments and metamorphoses, In: "Deformation Quantization", G. Halbout (ed.), IRMA Lectures Notes in Math. Phys., Vol. 1, Walter de Gruyter, Berlin, 2002, 9-54.

[18] D. Elworthy and D. Truman, Classical mechanics, the diffusion heat equation and the Schrödinger equation, J. Math. Phys. 22 (1981), 2144-2166.

[19] M. Gradinaru, F. Russo and P. Vallois, Generalized covariations, local time and Stratonovitch-Itô's formula for fractional Brownian motion, Ann. Probab. 31 (2003), 17721820.

[20] A. Hirshfeld, Deformation quantization in quantum mechanics and quantum field theory, In: "Geometry, Integrability and Quantization. IV", Mladenov I. and Naber G. (eds.), Coral Press, Sofia, 2003, 11-38.

[21] A. Hirshfeld and T. SChWARZWELler, Path integral quantization of the Poisson-Sigma model, Ann. Phys. (Leipzig), 9 (2000), 83-101.

[22] J. JACOD, "Calcul Stochastique et Problemes de Martingales", Lect. Notes. Math., Vol. 714, Springer, Heidelberg, 1975.

[23] J. R. KlAUdER and S. V. SHABANOV, An introduction to coordinate-free quantization and its application to constrained systems, In: "Mathematical Methods of Quantum Physics", C. C. Bernido (ed.), Gordon and Breach., Amsterdam, 1999, 117-131.

[24] M. Kontsevich, Deformation quantization of Poisson manifolds, Lett. Math. Phys., to appear.

[25] H. Kunita, "Stochastic Flows and Stochastic Differential Equations", Camb. Univ. Press, Cambridge, 1990.

[26] H. H. KUO, Diffusion and Brownian motion on infinite dimensional manifolds, Trans. Amer. Math. Soc. 159 (1972), 439-451.

[27] S. KuSUOKA, More recent theory of Malliavin Calculus, Sūgaku 5 (1992), 155-173.

[28] R. LÉANDre, Applications quantitatives et qualitatives du Calcul de Malliavin, In: "French-Japanese Seminar", Métivier M. and Watanabe S. (eds.). Lect. Notes. Math., Vol. 1322, Springer, Berlin, 1988, 109-123. English translation in: "Geometry of Random Motion", Durrett R. and Pinsky M. (eds.) Contem. Math. 73, A.M.S., Providence, 1988, 173-197.

[29] R. LÉANDRE, Cover of the Brownian bridge and stochastic symplectic action, Rev. Math. Phys. 12 (2000), 91-137.

[30] R. LÉANDRE, Analysis on loop spaces and topology, Math. Notes. 72 (2002), 212-229.

[31] R. LÉAndre, Stochastic Wess-Zumino-Novikov-Witten model on the torus, J. Math. Phys. 44 (2003), 5530-5568.

[32] R. LÉANDRE, Brownian cylinders and intersecting branes, Rep. Math. Phys. 52 (2003), 363-372.

[33] R. LÉANDRE, Markov property and operads, In: "Quantum Limits in the Second Law of Thermodynamics", I. Nikulov and D. Sheehan (eds.), Entropy, Vol. 6, 2004, 180-215.

[34] R. LÉAndRe, Brownian pants and Deligne cohomology, J. Math. Phys. 46 (2005).

[35] R. LÉANDRE, Bundle gerbes and Brownian motion, In: "Lie Theory and Application in Physics. V.", V. Dobrev and H. Doebner (eds.). World Scientific, Singapore, 2004, 343-352.

[36] R. LÉANDRE, Galton-Watson tree and branching loop, In: "Geometry, Integrability and Quantization. VI", I. Mladenov and A. Hirshfeld (eds.), Softek, Sofia, 2005, 276-284.

[37] R. LÉANDRE, Two examples of stochastic field theories, Osaka J. Math. 42 (2005), 353365.

[38] P. Malliavin, Stochastic Calculus of variation and hypoelliptic operators, In: "Stochastic Analysis", K. Itô (ed.), Kinokuyina, Tokyo, 1978, 155-263.

[39] P. A. Meyer, Flot d'une équation différentielle stochastique, In: "Séminaire de Probabilités. XV", Azéma J. and Yor M. (eds.), Lect. Notes. Math., Vol. 850, Springer, Heidelberg, 1981, 100-117.

[40] S. Molchanov, Diffusion processes and Riemannian geometry, Russian Math. Surveys 30 (1975), 1-63. 
[41] D. Nualart, "Malliavin Calculus and Related Topics", Springer, Heidelberg, 1997.

[42] D. NUALART and M. SANZ, Malliavin Calculus for two-parameter Wiener functionals, Z. Wahrsch. Verw. Gebiete 70 (1985), 573-590.

[43] V. PIPIRAS and M. TAQQU, Integration question related to fractional Brownian motion, Probab. Theory Related Fields 118 (2000), 251-291.

[44] H. K. Sugita, Positive generalized Wiener functions and potential theory over abstract Wiener spaces, Osaka J. Math. 25 (1988), 665-696.

[45] S. Watanabe, Stochastic analysis and its application Sūgaku 5 (1992), 51-71.

Institut de Mathématiques

Faculté des Sciences

Université de Bourgogne

21000 Dijon, France

Remi.leandre@u-bourgogne.fr 